\title{
Acupuncture Injection Combined with Electrokinetic Injection for Polydimethylsiloxane Microfluidic Devices
}

\author{
Ji Won Ha \\ Department of Chemistry, University of Ulsan, 93 Daehak-Ro, Nam-Gu, Ulsan 44610, Republic of Korea \\ Correspondence should be addressed to Ji Won Ha; jwha77@ulsan.ac.kr
}

Received 15 January 2017; Revised 9 February 2017; Accepted 12 February 2017; Published 23 February 2017

Academic Editor: Chih-Ching Huang

Copyright (C) 2017 Ji Won Ha. This is an open access article distributed under the Creative Commons Attribution License, which permits unrestricted use, distribution, and reproduction in any medium, provided the original work is properly cited.

\begin{abstract}
We recently reported acupuncture sample injection that leads to reproducible injection of nL-scale sample segments into a polydimethylsiloxane (PDMS) microchannel for microchip capillary electrophoresis. The advantages of the acupuncture injection in microchip capillary electrophoresis include capability of minimizing sample loss and voltage control hardware and capability of introducing sample plugs into any desired position of a microchannel. However, the challenge in the previous study was to achieve reproducible, pL-scale sample injections into PDMS microchannels. In the present study, we introduce an acupuncture injection technique combined with electrokinetic injection (AICEI) technique to inject pL-scale sample segments for microchip capillary electrophoresis. We carried out the capillary zone electrophoresis (CZE) separation of FITC and fluorescein, and the mixture of $10 \mu \mathrm{M}$ FITC and $10 \mu \mathrm{M}$ fluorescein was separated completely by using the AICEI method.
\end{abstract}

\section{Introduction}

Microchip capillary electrophoresis (CE) emerged in the early 1990 s as an interesting and novel approach in a variety of bioanalytical applications. For example, microchip CE has been widely used for the analysis of a variety of samples that include amino acids [1], peptides [2], proteins [3], and DNA fragments [4]. Microchip CE has many advantages of decreased analysis time, integrated sample processing, high portability, high throughput, minimal reagent consumption, low analysis cost, and so forth.

In microchip CE, sample injection is one of the most critical parameters for successful separation and analysis. In this regard, it is of great importance to develop sample injection methods that lead to well-defined sample plugs for high-performance microchip CE. The electrokinetic injection is the most common method to inject a sample solution in microchip-based CE. The electrokinetic injection method is based on the electroosmotic flow (EOF) generated by applying high voltages in a microchannel. In electrokinetic injection, tee [5, 6], cross [7], and double-tee [8] injectors have been used to dispense well-defined sample plugs into a separation channel. In addition, several electrokinetic injection modes have been developed, which includes gated $[9,10]$, pinched $[11,12]$, and floating [13].

However, all electrokinetic injection modes need inconvenient voltage programs for dispensing well-defined sample plugs and require complicated hard and software systems for chip operation. Furthermore, the amounts of sample loss are quite large compared to real injected amounts into separation channel. Recently, there have been many efforts to overcome the aforementioned limitations in electrokinetic injection [14-24]. Despite the recent efforts, it is still necessary to develop simpler and more cost-effective methods for sample injection on microchips.

Very recently, we developed an acupuncture injection technique to form the well-defined sample plugs in PDMS microchannels [24]. This technique enabled us to achieve reproducible injection of $3 \mathrm{~nL}$ sample plug into a microchannel. Microchip CE was performed by applying a single potential in the most simplified straight channel. The advantages of this acupuncture injection in microchip CE include capability of minimizing sample consumption and voltage control hardware, capability of serial injections of different samples into a 
same channel, and capability of introducing sample segments into any desired position of a microchannel. However, the challenge in the previous study was to achieve reproducible, pL-scale sample injections into PDMS microchannels.

In this paper, we present an acupuncture injection combined with electrokinetic injection (AICEI) for polydimethylsiloxane (PDMS) microchips. We demonstrate that the AICEI technique allows for the injection of pL-scale segments into a PDMS microchannel while minimizing the sample loss. A pL-scale sample plug was produced by a fast-dipping method, and the sample plug was then injected into a PDMS microchip by performing acupuncture on a channel with a homemade capillary needle and applying a high voltage to drive the sample plug.

\section{Experimental Section}

2.1. Chemicals and Materials. PDMS was purchased from Dow Corning (Midland, MI, USA) under the product name Sylgard 184. Microslides were purchased from VWR Scientific Inc. (West Chester, PA, USA). All reagents used in this paper were of analytical grade and were obtained from SigmaAldrich (St. Louis, MO, USA). All aqueous solutions were prepared by using water purified with a Milli-Q purifying system (Millipore, Milford, MA, USA). An injector composed of a micrometer, a syringe holder, and a translator was a part of a contact angle meter (MODEL: KRUSS G10) and was purchased from Marktech Co. (Seoul, Republic of Korea). 30 gauge-disposable needles were obtained from Becton Dickinson Co. (Franklin Lakes, NJ, USA).

2.2. Fabrication of PDMS Microchips. PDMS microchips in this paper were fabricated by photolithography replica molding method [25-28]. Channel networks were designed by using a computer aided design software package (AutoCAD; Autodesk, San Rafael, CA, USA). The network designs were converted into a sheet of mask. A $25 \mu \mathrm{m}$ thick film of a negative photoresist (SU-8 50; MicroChem, Newton, MA, USA) was spin-coated on a silicon wafer of $100 \mathrm{~mm}$ diameter (LG Siltron, South Korea), exposed to UV light through the mask, and then developed in propylene glycol methyl ether acetate (Aldrich, Milwaukee, WI, USA) to reveal a master with a positive relief pattern of the photoresist. The master was silanized by placing it in a vacuum desiccator for $2 \mathrm{~h}$ along with a vial containing a few drops of a silanizing agent, tricholoro(3,3,3-trifluoroproyl)silane (Aldrich). A $10: 1$ mixture of PDMS oligomer and cross-linking agent (Sylgard 184) was poured onto the master and then degassed under vacuum. After at least $3 \mathrm{~h}$ of curing at $75^{\circ} \mathrm{C}$, a PDMS replica was peeled from the master to yield the negative relief of channel networks. The reservoirs were defined by punching holes at the dead ends of channels. A flat slab of PDMS was made similarly by casting the Sylgard 184 mixture against a silanized silicon wafer. A PDMS replica and a flat slab were rinsed in methanol and dried under a stream of nitrogen. The surfaces of the two PDMS pieces, which will face each other, were oxidized by corona discharge generated from a Tesla coil (BD-10A; Electro Technic Products, Chicago,

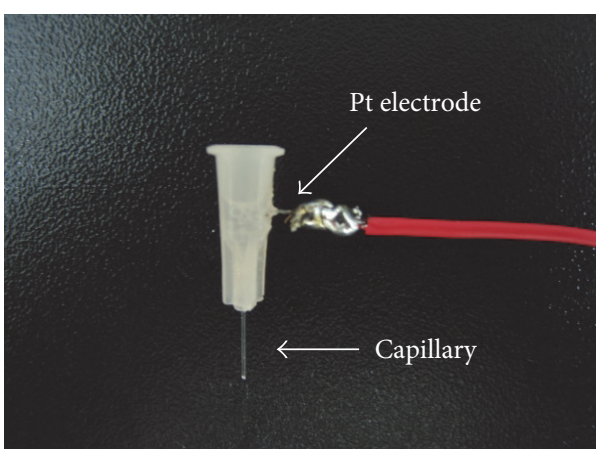

FIgURE 1: A photographic image of a homemade capillary needle designed for AICEI method. The tip was grinded into a slant face. $\mathrm{Pt}$ electrode was inserted and firmly fixed into the cone of a capillary needle.

IL, USA) for $2 \mathrm{~min}$. Immediately after the treatment, the two pieces were brought into conformal contact, and a complete irreversible seal was accomplished spontaneously in 3 h. Figure S1 (in Supplementary Material available online at https://doi.org/10.1155/2017/7495348) shows a photographic image of PDMS microchips used for the CZE experiment in this study. The channel length from reservoir 1 to reservoir 2 was $38 \mathrm{~mm}$, while the channel width and height was $100 \mu \mathrm{m}$.

2.3. Fabrication of a Capillary Needle. In the present study, we employed a homemade capillary needle for electrically driven acupuncture injection (Figure 1). The outer and inner diameters of the capillary needle were $300 \mu \mathrm{m}$ and $100 \mu \mathrm{m}$, respectively. In this sample injection method, the plastic cone of a capillary needle serves as a reservoir where a buffer solution is contained. Therefore, a Pt electrode was inserted into the plastic cone and was firmly fixed with an adhesive. The tip of a capillary needle was modified and grinded into a slant face.

2.4. Apparatus and Instrumentation. Electrokinetic injection and separation were controlled by using a computercontrolled high-voltage supplying system equipped with a high-voltage power supply (MP5; Spellman High Voltage Electronics, Plainview, NY, USA), a high-voltage relay (K45C332; Kilovac, Santa Barbara, CA, USA), and a homemade voltage dividing system. A LabVIEW (National Instruments, Austin, TX, USA) program written in-house and a multifunction I/O board (Lab-PC-1200; National Instruments) were used for instrument control and data acquisition.

For laser-induced fluorescence (LIF) detection, a laser beam was incident on the chip at an angle of $45^{\circ}$ and focused on near the end of the separation channel with a planoconvex fused-silica lens (100 mm focal length; CVI Laser Optics \& Coatings, Alibuquerque, NM, USA). The fluorescence light was collected from below the chip with a 10x microscope objective (numerical aperture, 0.3; Nikon, Japan) and directed to a photomultiplier tube (HC12001; Hamamatsu, Bridgewater, NJ, USA) through a $0.5 \mathrm{~mm}$ pinhole located at the image plane of the objective lens. Band pass filters (10 nm bandwidth; CVI Laser Optics \& Coatings) 


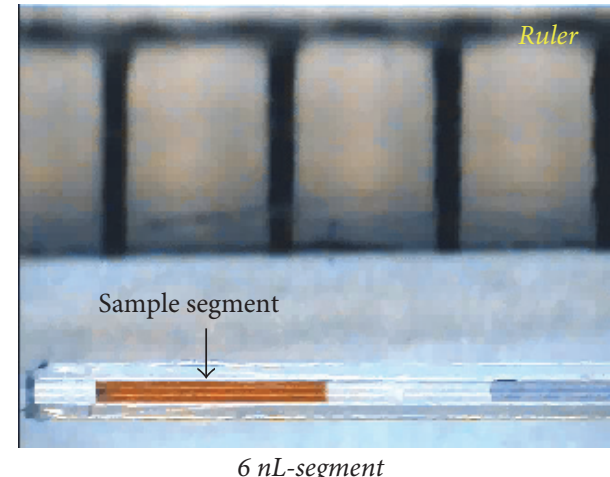

(a)

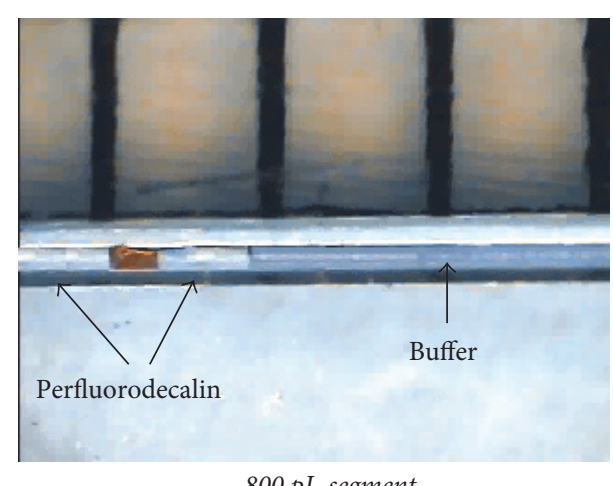

800 pL-segment

(b)

FIGURE 2: (a) CCD image of a sample segment (red-color) in between perfluorodecalin plugs. The loaded volume was calculated to be $\sim 6 \mathrm{~nL}$. A buffer solution was mixed with blue-ink for clearer demonstration. (b) CCD image of a sample segment (red-color) with the loaded volume of $800 \mathrm{pL}$.

were used for eliminating the scatter of the excitation line of the argon ion laser (Lexel 95; Lexel Laser, Fremont, CA, USA).

\subsection{Capillary Zone Electrophoresis. Before performing the} CZE experiment, the channel networks were washed with methanol and deionized water for $10 \mathrm{~min}$. A $4 \mathrm{mM}$ boric acid and $20 \mathrm{mM}$ Tris(hydroxymethyl) aminomethane buffer (TRIS) mixture ( $\mathrm{pH} 9.0$ ) containing $10 \mathrm{mM}$ sodium dodecyl sulfate (SDS) was used as electrophoretic medium. A mixture of $10 \mu \mathrm{M}$ FITC and $10 \mu \mathrm{M}$ fluorescein was used as the sample. CZE separation was performed using the straight channel microchip shown in Figure S1. In this method, only a single potential was applied at two reservoirs for CZE separation. In this study, sample was detected by LIF detection method in which an argon ion laser (Lexel 95; Lexel Laser, Fremont, CA, USA) was employed as the excitation source.

\section{Results and Discussion}

The generation of well-defined sample plugs is critical for high-performance CE separation and analysis. In this study, the sampling was carried out by a fast-dipping method based on a capillary action. More specifically, the capillary needle filled with a buffer solution (50 $\mathrm{mM}$ phosphate) was sequentially dipped into perfluorodecalin and sample solutions in order to make a sample segment inside a needle (Figure S2). The reason for using perfluorodecalin immiscible with aqueous phase is to avoid the diffusion and dilution of sample segments to be injected into a separation channel. Figure S3 shows the effect of perfluorodecalin on the diffusion of a sample plug. Figure S3A is a CCD image of a sample plug with perfluorodecalin at both ends of the sample plug. We observed that the diffusion of a sample segment into the buffer solution was avoided in the presence of perfluorodecalin segments. However, the dilution was observed in the absence of perfluorodecalin at both ends of the sample plug. Therefore, this dipping method allowed us to produce a welldefined aqueous sample plug in between perfluorodecalin segments inside the capillary needle.
As a next step, it is important to determine the amounts of sample loaded into the capillary needle by the fast-dipping method. In this study, the length of a sample plug was measured (or estimated) by using a ruler, and then we determined the loaded volume by calculating the volume of a cylinder. Figure 2 shows sample segments (red-colour) loaded into the capillary needle. In Figure 2(a), the loaded volume was calculated to be about $6 \mathrm{~nL}$. We further tried to reduce the volume of a sample plug loaded into the capillary needle by the fast-dipping method, and we could push it down to $800 \mathrm{pL}$ as shown in Figure 2(b). This indicates that pL-scale sample segments can be generated in the capillary needle by the fast-dipping method. Therefore, it should be noted that we developed a new way to form well-defined, pL-scale sample segments in a capillary needle without a diffusion problem.

The next crucial step is to dispense the sample plug into a PDMS microchip. Our idea is to introduce the sample plug by applying acupuncture on a microchannel and applying a high voltage for inducing EOF. More concretely, Figure 3 depicts a schematic of AICEI method. Initially, the homemade capillary needle position is adjusted in the horizontal plane with the assistance of a CCD camera (Figure 3(a)). Then, the PDMS microchannel is carefully acupunctured vertically with the capillary needle (Figure 3(b)). After applying acupuncture, the sample plug is introduced into a separation channel by applying a high voltage to generate EOF as shown in Figure 3(b).

Now, it is important to experimentally verify the approach described in Figure 3. In the present study, we developed an instrumental setup to realize the AICEI method (Figure S4). The instrument consists of a high voltage supplier, a CCD camera, a chip holder, and an injector. The injector, a part of a contact angle meter, is composed of a micrometer, a syringe holder, and a translator (Figure S4B). After setting up the system, a microsyringe with a sample plug in the capillary needle was fixed to the holder of injector. We then tried to inject sample segments into a PDMS microfluidic channel by the AICEI method in Figure 3. Figure 4 shows consecutive 


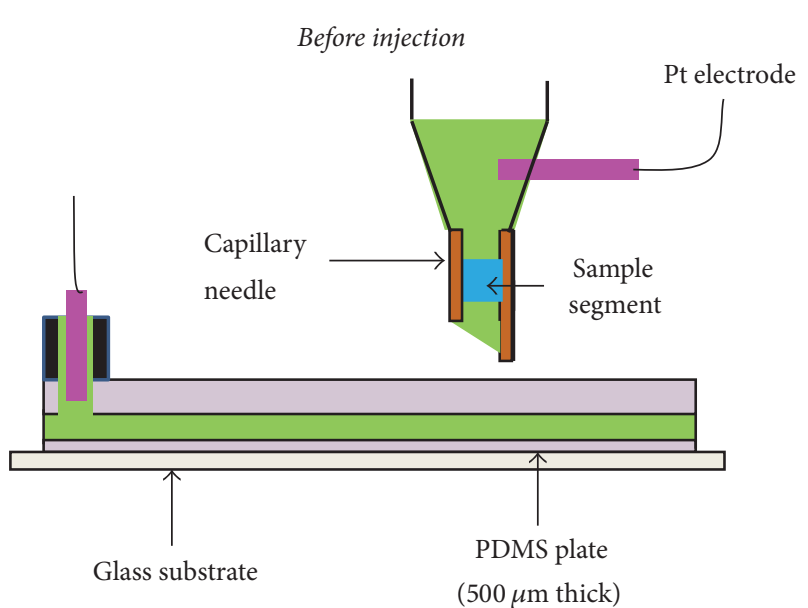

(a)

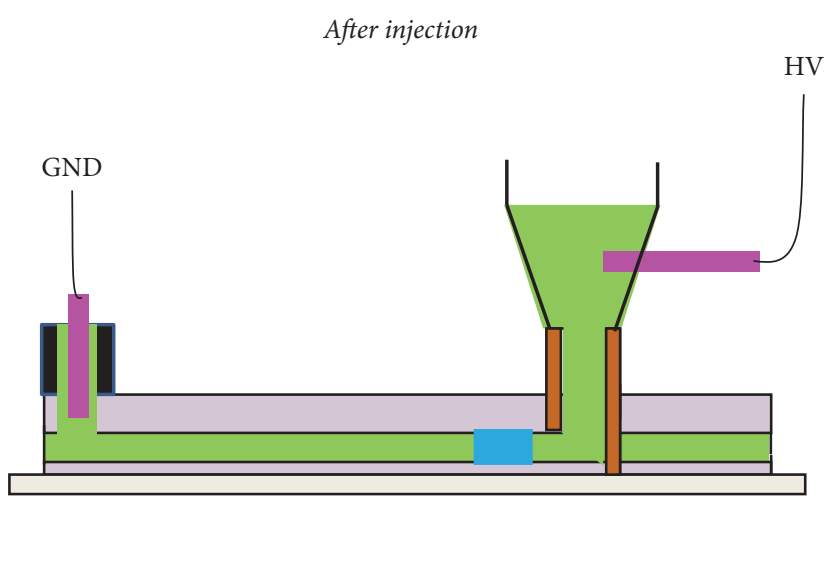

(b)

FIGURE 3: Schematic diagram to show the AICEI technique. (a) Before injection: a homemade capillary needle position is adjusted in the horizontal plane. (b) After injection: a sample segment is injected by applying acupuncture on a channel and applying a high voltage (single potential, $1 \mathrm{kV})$.

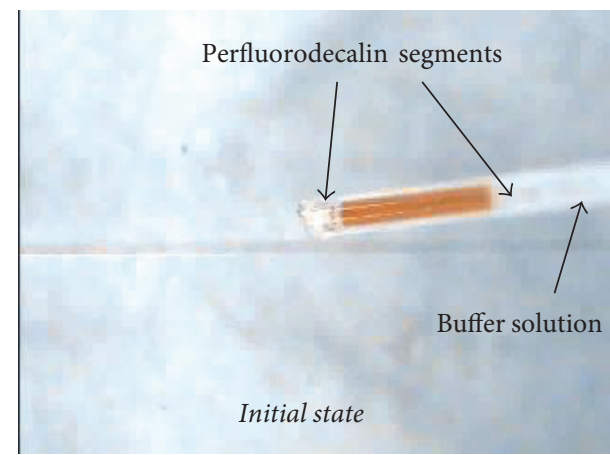

(a)

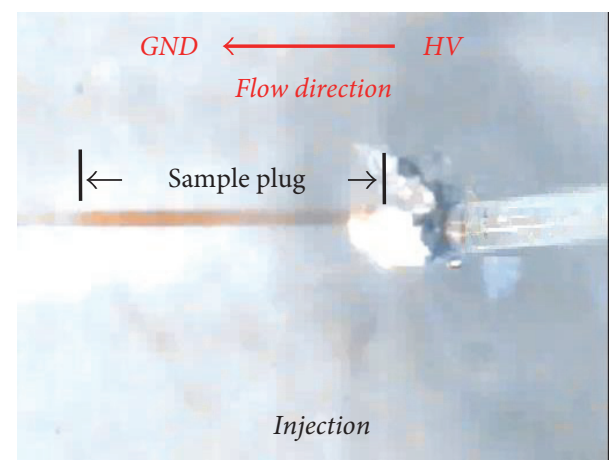

(c)

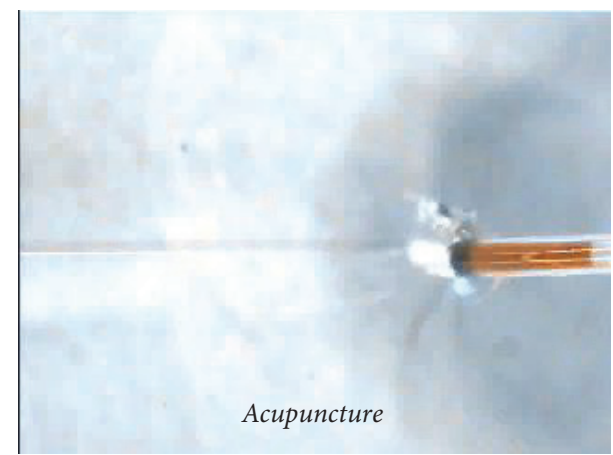

(b)

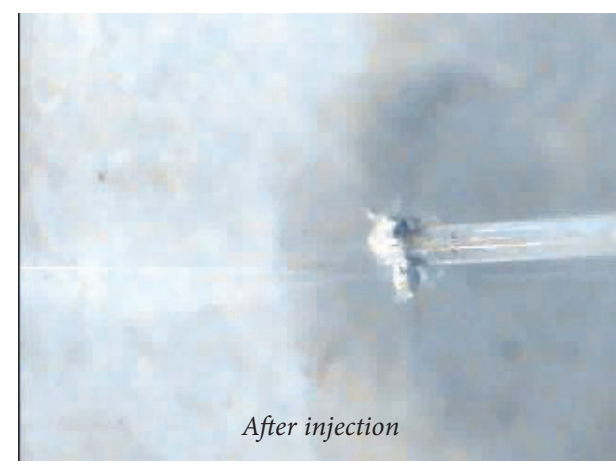

(d)

FIGURE 4: Consecutive CCD images recorded at the different stages of sample injection using the AICEI method from the top view. (a) Initial state, (b) acupuncture, (c) sample injection, and (d) after injection.

CCD images of introducing a sample plug using the AICEI method from the top view. Initially, the needle position in the horizontal plane was adjusted with the assistance of a CCD camera (Figure 4(a)). We then inserted the capillary needle into the microchannel until it touches at the surface of the glass substrate (Figure 4(b)). After this, we applied a high voltage $(1 \mathrm{kV})$ to drive the red-coloured sample plug into the microchannel (Figure 4(c)). Finally, a sample plug was flowed in the microchannel (Figure 4(d)). At the end of experiment, the capillary needle was pulled out carefully from the microchip. Therefore, we experimentally confirmed that the AICEI technique can be used to inject well-defined 


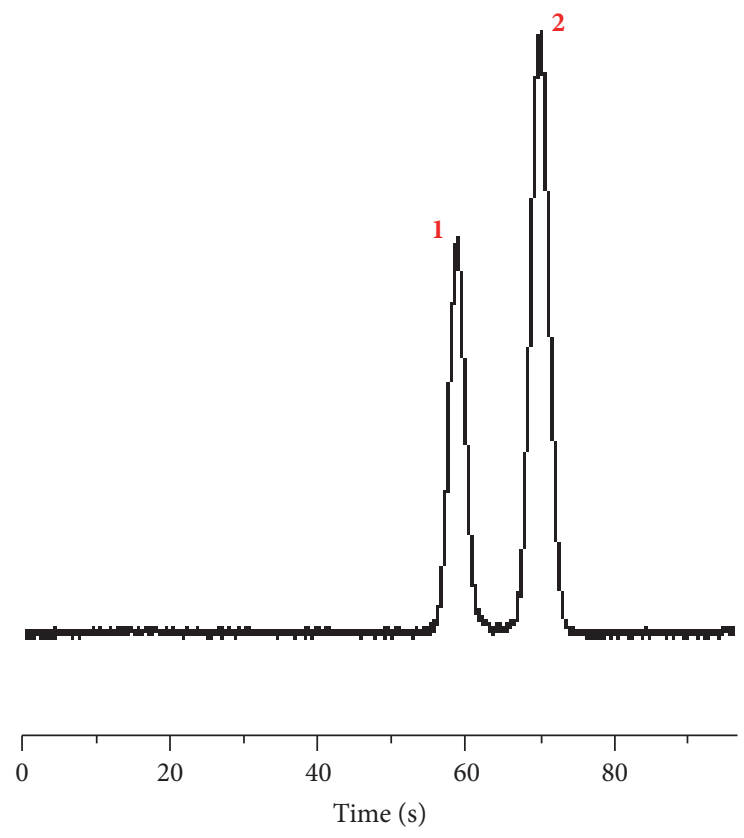

FIGURE 5: CZE separation of $10 \mu \mathrm{M}$ FITC (peak 1) and $10 \mu \mathrm{M}$ fluorescein (peak 2) using the AICEI method.

sample plugs into a PDMS microchip. Furthermore, this AICEI method allows us to achieve pL-scale sample injection, which was not achieved by the acupuncture injection in our previous study [24].

Last, it is necessary to demonstrate that the AICEI method can be directly used for the microchip CE separation. We therefore performed CZE separation of FITC and fluorescein by using the AICEI method. As shown in the electropherogram (Figure 5), the mixture of $10 \mu \mathrm{M}$ FITC and $10 \mu \mathrm{M}$ fluorescein was separated completely by the AICEI method. Compared to typical gated and pinched mode sample injection, this AICEI method requires only a single potential application, which is greater advantage. Furthermore, the AICEI method provides more advantages including capability of minimizing the sample loss, capability of injecting samples into any position of a channel, and ease in fabricating microchips.

\section{Conclusions}

In summary, a new acupuncture injection technique in combination with electrokinetic injection is introduced for polydimethylsiloxane (PDMS) microfluidic devices. This acupuncture injection technique has several advantages that include capability of minimizing sample consumption and voltage control hardware, capability of serial injections of different sample solutions into a same microchannel, and capability of introducing sample segments into any position of a microchannel. In this work, we present that pL-scale sample plugs can be injected into a PDMS microfluidic device by performing acupuncture on a channel with a homemade capillary needle and applying a high voltage to drive the sample plugs for microchip CE separation ad analysis.
Furthermore, we demonstrate that the AICEI method can be used for CZE separation in the most simplified straight channel with a single potential.

\section{Competing Interests}

The author declares that they have no competing interests.

\section{Acknowledgments}

This work was supported by the National Research Foundation of Korea (NRF) grant funded by the Korea government (MSIP) (no. 2015R1C1A1A01052995).

\section{References}

[1] J. Qiu, L. Wang, R. Liang, and J. Wang, "Microchip CE analysis of amino acids on a titanium dioxide nanoparticlescoated PDMS microfluidic device with in-channel indirect amperometric detection," Electrophoresis, vol. 30, no. 19, pp. 3472-3479, 2009.

[2] B. A. Fogarty, N. A. Lacher, and S. M. Lunte, "Microchip capillary electrophoresis: application to peptide analysis," in Microchip Capillary Electrophoresis, vol. 339 of Methods in Molecular Biology, pp. 159-186, Humana Press, 2006.

[3] J. S. Creamer, N. J. Oborny, and S. M. Lunte, "Recent advances in the analysis of therapeutic proteins by capillary and microchip electrophoresis," Analytical Methods, vol. 6, no. 15, pp. 54275449, 2014.

[4] Y. Shi, P. C. Simpson, J. R. Scherer et al., "Radial capillary array electrophoresis microplate and scanner for high-performance nucleic acid analysis," Analytical Chemistry, vol. 71, no. 23, pp. 5354-5361, 1999.

[5] A. Manz, D. Harrison, E. M. Verpoorte et al., "Planar chips technology for miniaturization and integration of separation techniques into monitoring systems," Journal of Chromatography A, vol. 593, no. 1-2, pp. 253-258, 1992.

[6] D. J. Harrison, A. Manz, Z. Fan, H. Luedi, and H. M. Widmer, "Capillary electrophoresis and sample injection systems integrated on a planar glass chip," Analytical Chemistry, vol. 64, no. 17, pp. 1926-1932, 1992.

[7] D. J. Harrison, K. Fluri, K. Seiler, Z. Fan, C. S. Effenhauser, and A. Manz, "Micromachining a miniaturized capillary electrophoresis-based chemical analysis system on a chip," Science, vol. 261, no. 5123, pp. 895-897, 1993.

[8] C. S. Effenhauser, A. Paulus, A. Manz, and H. M. Widmer, "High-speed separation of antisense oligonucleotides on a micromachined capillary electrophoresis device," Analytical Chemistry, vol. 66, no. 18, pp. 2949-2953, 1994.

[9] X. Wang, Y. Zhu, and Q. Fang, "Valveless gated injection for microfluidic chip-based liquid chromatography system with polymer monolithic column," Journal of Chromatography A, vol. 1246, pp. 123-128, 2012.

[10] B. W. Wenclawiak and R. J. Püschl, "Sample injection for capillary electrophoresis on a micro fabricated device/on chip CE injection," Analytical Letters, vol. 39, no. 1, pp. 3-16, 2006.

[11] S. C. Jacobson, R. Hergenroder, L. B. Koutny, R. J. Warmack, and J. M. Ramsey, "Effects of Injection Schemes and Column Geometry on the Performance of Microchip Electrophoresis Devices," Analytical Chemistry, vol. 66, no. 7, pp. 1107-1113, 1994. 
[12] L. Zhang, X. Yin, and Z. Fang, "Negative pressure pinched sample injection for microchip-based electrophoresis," Lab on a Chip, vol. 6, no. 2, pp. 258-264, 2006.

[13] J. Khandurina, T. E. McKnight, S. C. Jacobson, L. C. Waters, R. S. Foote, and J. M. Ramsey, "Integrated system for rapid PCR-based DNA analysis in microfluidic devices," Analytical Chemistry, vol. 72, no. 13, pp. 2995-3000, 2000.

[14] J. Li, C. Liu, Z. Xu et al., "A bio-inspired micropump based on stomatal transpiration in plants," Lab on a Chip, vol. 11, no. 16, p. 2785, 2011.

[15] U. Kalsoom, R. M. Guijt, M. C. Boyce, A. T. Townsend, R. Haselberg, and M. C. Breadmore, "Direct electrokinetic injection of inorganic cations from whole fruits and vegetables for capillary electrophoresis analysis," Journal of Chromatography A, vol. 1428, pp. 346-351, 2016.

[16] M. Blas, N. Delaunay, and J.-L. Rocca, "Electrokinetic-based injection modes for separative microsystems," Electrophoresis, vol. 29, no. 1, pp. 20-32, 2008.

[17] Y. Cong, S. Katipamula, T. Geng, S. A. Prost, K. Tang, and R. T. Kelly, "Electrokinetic sample preconcentration and hydrodynamic sample injection for microchip electrophoresis using a pneumatic microvalve," Electrophoresis, vol. 37, no. 3, pp. 455462, 2016.

[18] I. K. Kiplagat, T. K. Doan, P. Kubáň, and P. Boček, “Trace determination of perchlorate using electromembrane extraction and capillary electrophoresis with capacitively coupled contactless conductivity detection," ELECTROPHORESIS, vol. 32, no. 21, pp. 3008-3015, 2011.

[19] X. Bai, H. J. Lee, J. S. Rossier et al., "Pressure pinched injection of nanolitre volumes in planar micro-analytical devices," Lab on a Chip-Miniaturisation for Chemistry and Biology, vol. 2, no. 1, pp. 45-49, 2002.

[20] L. Qi, X. Yin, L. Zhang, and M. Wang, "Rapid and variablevolume sample loading in sieving electrophoresis microchips using negative pressure combined with electrokinetic force," Lab on a Chip, vol. 8, no. 7, pp. 1137-1144, 2008.

[21] T. Hirokawa, Y. Takayama, A. Arai, and Z. Xu, "Study of a novel sample injection method (floating electrokinetic supercharging) for high-performance microchip electrophoresis of DNA fragments," ELECTROPHORESIS, vol. 29, no. 9, pp. 1829-1835, 2008.

[22] V. Sahore, S. Kumar, C. I. Rogers, J. K. Jensen, M. Sonker, and A. T. Woolley, "Pressure-actuated microfluidic devices for electrophoretic separation of pre-term birth biomarkers," Analytical and Bioanalytical Chemistry, vol. 408, no. 2, pp. 599607, 2016.

[23] H. Zhang, J. Gavina, and Y. Feng, "Understanding mechanisms of pressure-assisted electrokinetic injection: application to analysis of bromate, arsenic and selenium species in drinking water by capillary electrophoresis-mass spectrometry," Journal of Chromatography A, vol. 1218, no. 20, pp. 3095-3104, 2011.

[24] J. W. Ha and J. H. Hahn, "Acupuncture sample injection for microchip capillary electrophoresis and electrokinetic chromatography," Analytical Chemistry, vol. 88, no. 9, pp. 46294634, 2016.

[25] A. Kumar, N. L. Abbott, H. A. Biebuyck, E. Kim, and G. M. Whitesides, "Patterned self-assembled monolayers and mesoscale phenomena," Accounts of Chemical Research, vol. 28, no. 5, pp. 219-226, 1995.

[26] Y. Xia, E. Kim, X.-M. Zhao, J. A. Rogers, M. Prentiss, and G. M. Whitesides, "Complex optical surfaces formed by replica molding against elastomeric masters," Science, vol. 273, no. 5273, pp. 347-349, 1996.

[27] D. Qin, Y. Xia, J. A. Rogers, R. J. Jackman, X. Zhao, and G. M. Whitesides, "Microfabrication, microstructures and microsystems," in Microsystem Technology in Chemistry and Life Science, vol. 194 of Topics in Current Chemistry, pp. 1-20, Springer Berlin Heidelberg, Berlin, Germany, 1998.

[28] G. M. Whitesides, E. Ostuni, S. Takayama, X. Jiang, and D. E. Ingber, "Soft lithography in biology and biochemistry," Annual Review of Biomedical Engineering, vol. 3, no. 1, pp. 335-373, 2001. 

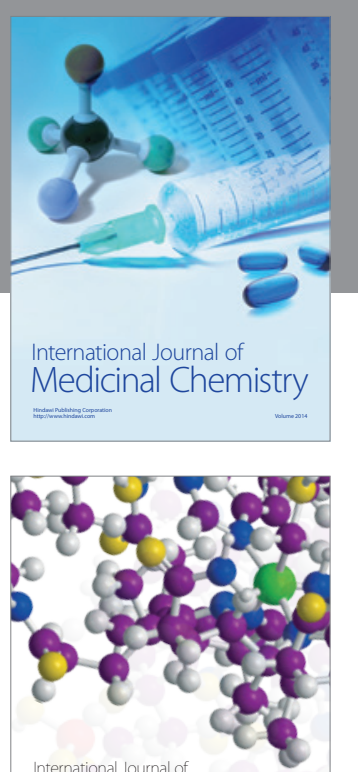

Carbohydrate Chemistry

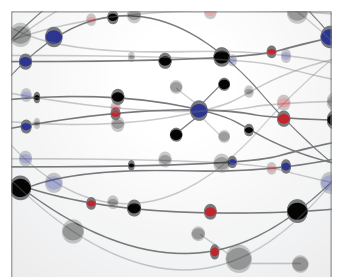

The Scientific World Journal
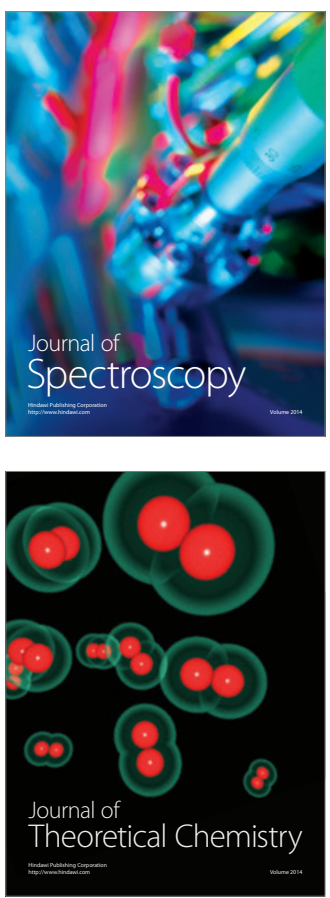
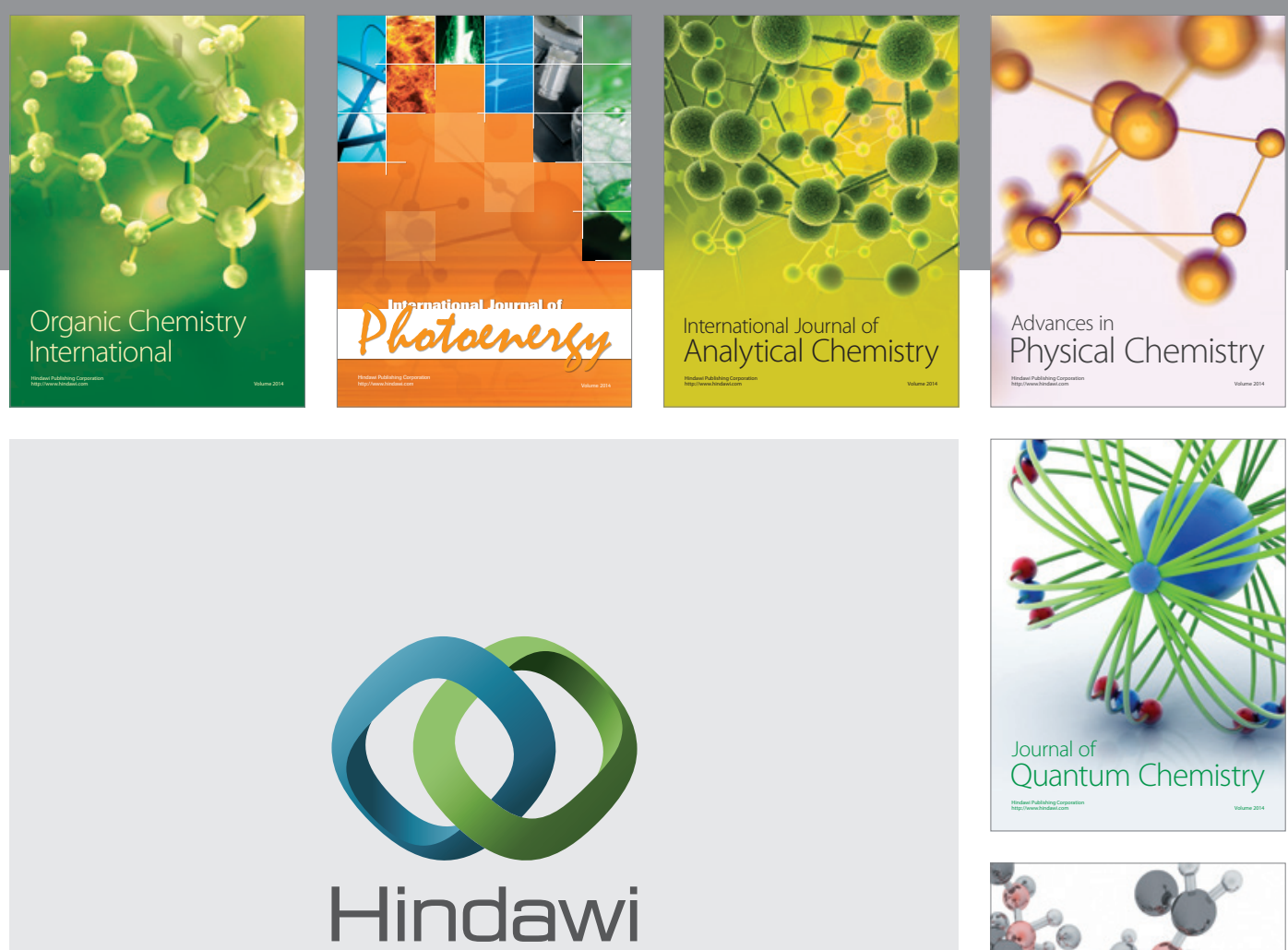

Submit your manuscripts at

https://www.hindawi.com

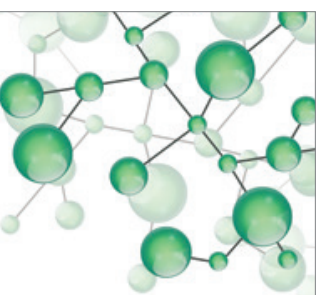

International Journal of

Inorganic Chemistry
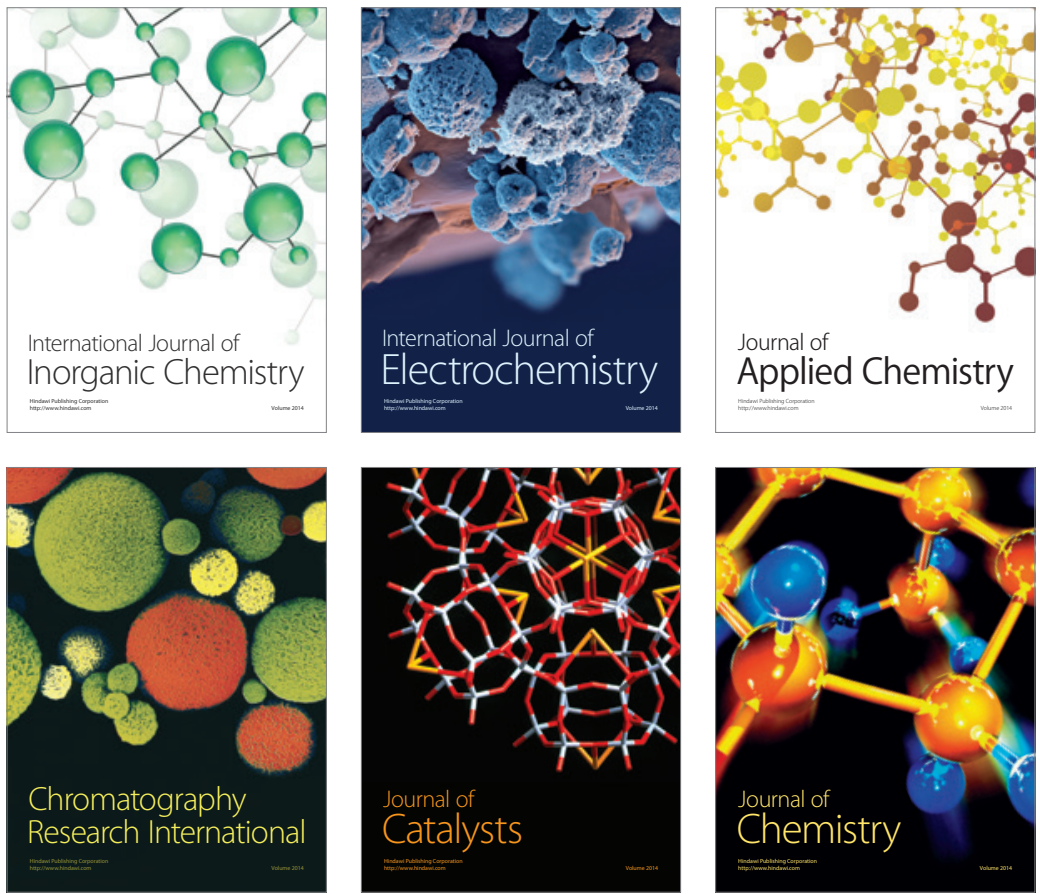

Journal of

Applied Chemistry
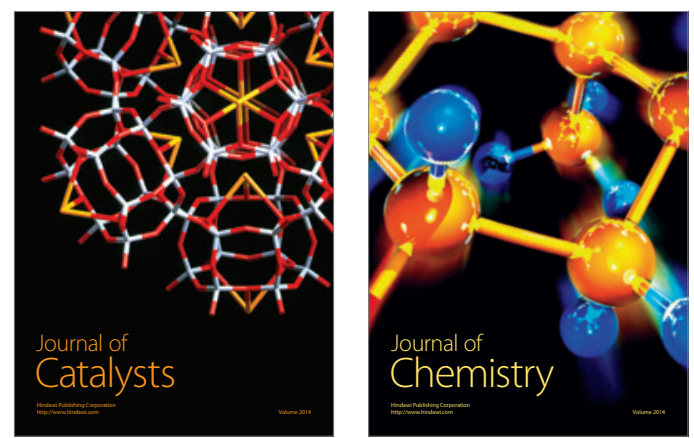
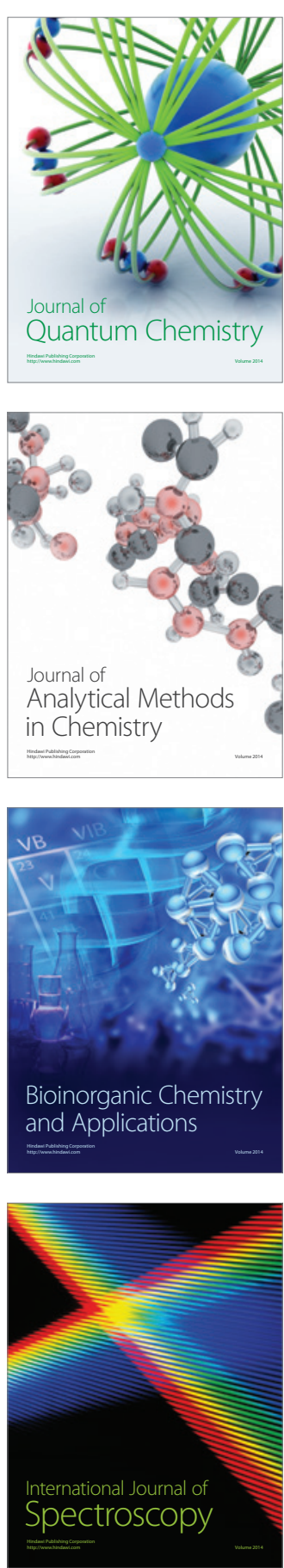